\title{
BAYESIAN AND MULTIVARIATE AUTOREGRESSIVE
}

\author{
Dr. Amaal Sadeq Hamoodi \\ Mathematics department, College of education \\ Mustansiriya university,Baghdad, Iraq \\ damal.sadiq@gmail.com
}

\section{Abstract}

This study related the Bayesian identification of the multivariate moving average processes using the bayes theorem, which combined the approximate likelihood function and a normal matrix-wishart prior density as a conjugate prior or a Jeffrey,'s as a vague prior, this combination lead to the posterior distribution in order to reach the marginal posterior distribution. We use the approximation technique to reach to the approximated likelihood function because The Main problem with the exact Bayesian analysis of $\operatorname{VARMA}(\mathrm{p}, \mathrm{q})$ model is that there is no analytically form for the likelihood function. Because the residuals are not quadratic function of the model parameters, in other words $\operatorname{VARMA}(\mathrm{p}, \mathrm{q})$ model is non liner in its coefficients in the $\operatorname{VMA}(q)$ part. The Bayesian identification approach is called the direct technique; this technique is dealing with marginal posterior probability mass function of the model order, is developed in a suitable form.

Then , one may find out the posterior probabilities over the grid of the order,and select the order with the highest probability to solve the identified problem, the multivariate autoregressive moving average in the Bayesian approaches is not developed yet, and also is not explored yet with any kind of analysis.

Keyword : approximation technique, identification parameters, bayes, multivariate autoregressive, direct technique.

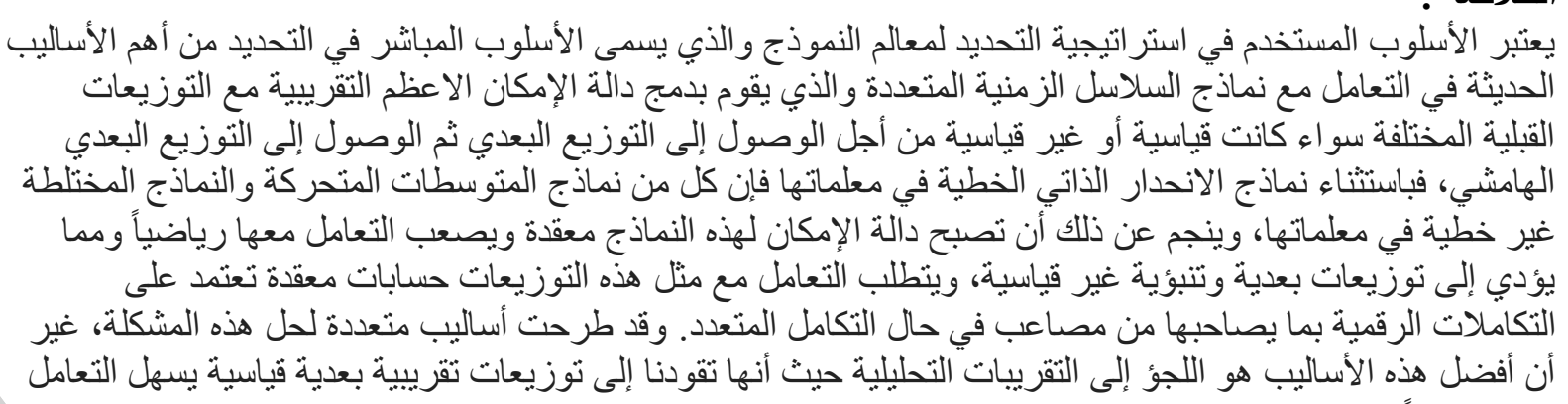

\section{INTRODUCTION}

The Time series model building protocol consists of four phases, which is start with identification, estimation, diagnostic check and end with forecast, the first phase which is identification is considered to be an important phase in which we are trying to estimating the order of $p$ and $q$ of the models such as $\operatorname{AR}(p), \operatorname{MA}(q)$, and ARMA(p,q). The effectiveness of the identification technique affects the precision of the forecasting process, which is the final objective of Times series analysis, moreover the perfect identification help to avoid estimation difficulties arising in such a models having redundant parameters.

In order to achieve the identification goal we have three approaches. The first one is Box- 
Jenkins methodology (See Box-Jenkins, 2016), they proposed an behavior investigation procedure in which both the sample autocorrelation function (ACF) and sample partial autocorrelation function (PACF) are used to decide which model to select from the set of $\operatorname{ARMA}(p, q)$ models. Then examining the plots of these functions using the theoretical (ACF) and (PACF) that have a characteristics pattern to determine the orders of the models, the problem with Box-Jenkins approach is the subjectivity of the method in comparing the sample (ACF) and (PACF) with the theoretical counterparts to decide which model to choose, moreover the difficulty in finding the identified model in the case of the mixed models since the case the sample (ACF) and (PACF) cannot give a clear cut about the orders of the model that should be identified and then some diagnostic test must be used, Finally we can say that this technique depends on the researcher's experience .

The second one of the identification approaches is called the automatic identification, unlike box-Jenkins approach, the decision is taken automatically without any human involvement in the model process , many method sorcriteria 'swecan defined, such as the residual variance criterion (RVC) method to identify $A R(p)$, and then used to identify MA(q) and $\operatorname{ARMA}(\mathrm{p}, \mathrm{q})$, also we have(Akiaka,2019) for propose the final prediction error (FPE) approach for (AR), also he propose the adjusted information criteria (AIC) criterion, and (BIC) which considered as the Bayesian criterion, which considered be a Bayesian modification criterion for (AIC), also (Schwarz,2018) introduced Schwarz criterion (SIC) to identify an $\operatorname{ARMA}(\mathrm{p}, \mathrm{q})$.

The third one is applying the Bayesian approach, which implies the usage of Bayes theorem concepts and terms such as, likelihood function and prior distributions which include non-informative (vague) prior density function as Jeffrey's one, or a proper distribution which preferred to be a conjugate prior densities, conjugate prior densities have the property that the prior and posterior densities both belong to the same parametric family such as, normalgamma prior in the unvariate level and normal matrix- wishart in the multivariate version, and finally posterior distribution as a Bayesian tool for the estimation phase.

The Bayesian time series analysis considered to be in a developing process, for well comprehend causes; the all Bayesian researches was focused and concentrated in the (AR) model and a few researches with the (MA) model or to the (ARMA) model, this avoid in the Bayesian literature is because the complication of the likelihood assignment, of the (MA) processes and also the (ARMA) processes, (Zellner,2011) starts the Bayesian analysis of time series witt. the autoregressive and distributed lag models, (Newbold,2013) used the Taylor's extension, for the remaining to develop very close to actual Bayesian analysis for the coefficients of the convey function models for the Bayesian identification, there is no Bayesian solution for the identification problem and it's still under development. The Main problem with the exact Bayesian analysis of $\operatorname{VARMA}(\mathrm{p}, \mathrm{q})$ model is that there is no analytic form for the likelihood function in the (VMA) part, Because the residuals are not quadratic function of the model parameters, in other words $\operatorname{VMA}(q)$ part is non liner in its coefficients. The researchers deals with $\operatorname{VARMA}(\mathrm{p}, \mathrm{q})$ in $\operatorname{VMA}(\mathrm{q})$ process part problem via many approaches to study the likelihood function. The used approach in our study is to use the approximation method to deliver the approximating $\operatorname{VARMA}(\mathrm{p}, \mathrm{q})$ likelihood function.

The foundation of this method is to approximate the likelihood function by replacing the exact residuals by their least square estimates. Using this approximation, we will develop the posterior distribution at the indirect Bayesian identification technique.

The main aim of this study is to manage the identification problem of (VARMA)(p,q) model from the Bayesian approach of identification, namely the direct approaches.

\section{VECTOR AUTOREGRESSIVE MOVING A VERAGE MODEL PROCESSES.}

$$
\text { Let }(\mathrm{t}) \text { be a sequence of integer, } \mathrm{p} \in(0,1,2, \ldots .), \mathrm{q} \in(0,1,2, \ldots .), \mathrm{k} \in(1.2, \ldots .),(\mathrm{y}(\mathrm{t})) \mathrm{a}
$$


sequence of $\mathrm{k} \mathrm{X} 1$ real observable random vectors, and a kpxk unknown matrix of a real constants, $\boldsymbol{a} \boldsymbol{k q} \boldsymbol{x} \boldsymbol{k}$ unknown matrix of a real constants, and $\varepsilon(t)$ a sequence of independent and normally distributed $\mathrm{kx} 1$ unobservable random vectors with zero mean and $\mathrm{k} \mathrm{x} \mathrm{k}$ unknown precision matrix $\mathrm{T}$, then the $\mathrm{k}$-variate autoregressive moving average process of order $(\mathrm{p}, \mathrm{q})$ using matrix notation is realize for $\mathrm{n}$ observed vectors as: $y(n x k)=Z(n x k(p+q)) \Gamma(k(p+q) x k)+U(n x k)$

Where:

$$
\begin{aligned}
& y(n x k)=(\mathrm{y}(1) y(2) \ldots \ldots \ldots . . \quad \mathrm{y}(n)]^{\prime}
\end{aligned}
$$

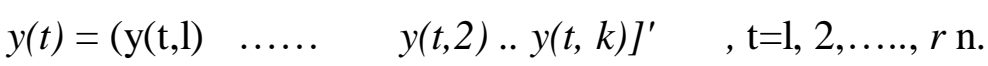$$
\mathrm{Z}_{(\mathrm{nXk}(\mathrm{p}+\mathrm{q}))}=\left[\begin{array}{c}
Z^{\prime}(0) \\
Z^{\prime}(1) \\
\cdots \\
Z^{\prime}(\mathrm{n}-1)
\end{array}\right]
$$$$
\Gamma_{(\mathrm{k}(\mathrm{p}+\mathrm{q}) \mathrm{xk})}=\left[\begin{array}{cccc}
\varnothing(1,1) & \varnothing(1,2) & \ldots & \varnothing(1, k) \\
\varnothing(2,1) & \varnothing(2,2) & \ldots & \varnothing(2, k) \\
\ldots & \ldots & & \ldots \\
\varnothing(k p, 1) & \varnothing(k p, 2) & \ldots & \varnothing(k p, k) \\
\theta(1,1) & \theta(1,2) & \ldots & \theta(1, k) \\
\theta(2,1) & \theta(2,2) & \ldots & \theta(2, k) \\
\ldots & \ldots & & \ldots \\
\theta(k q, 1) & \theta(k q, 2) & \ldots & \theta(k q, k)
\end{array}\right]=\left[\begin{array}{c}
\Phi_{k p x k} \\
\Theta_{k q x k}
\end{array}\right]
$$

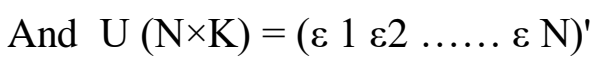

Where $\varepsilon(\mathrm{t})=\varepsilon[(\mathrm{t} .1) \varepsilon(\mathrm{t} .2) \ldots . . \varepsilon(\mathrm{t} . \mathrm{k})]^{\prime} \quad, \mathrm{t}=1,2, \ldots \ldots, \mathrm{n}$

\section{THE DIRECT IDENTIFICATION OF VARMA MODELS}

the multivariate autoregressive process (VAR). The version of the multivariate moving average model (VARMA)(p,q) in the direct Bayesian approach in coincidence phase of the multivariate times series has not been developed yet, direct technique propose to choose the order of a multivariate moving average process (VARMA)(p,q) on the basis of $n$ vectors observations. In this technique, the orders of the model are considered random variables and then the technique drives the posterior mass function for the chosen orders. After that, the posterior probabilities are computed to choose the orders with maximum probability as point estimates for the orders.

The technique also assumes that Conditioning on the first $\mathrm{p}$ observed vectors and assuming that $\varepsilon(p-1)=\ldots=\varepsilon(P-q-1)=0$.

The main aim of this part is to develop the posterior probability mass function of the order $\mathrm{p}$ and $\mathrm{q}$ under the conditions summarized above, In regularly to do that we need to combine three terms.

First, the likelihood function of the k-varaite moving average process of order q VARMA $(\mathrm{p}, \mathrm{q})$, which can be written as: 


$\mathrm{L}\left(\Gamma, \mathrm{T} / \mathrm{S}_{(\mathrm{n})}\right) \propto(2 \pi)^{-k(n-p) / 2}|T|^{n-p / 2} \exp \left(-\frac{1}{2} \operatorname{tr} \sum_{t=p+1}^{n}\left(\varepsilon^{\prime}(t) T \varepsilon(t)\right)\right)$
$\mathrm{L}\left(\Gamma, \mathrm{T} / \mathrm{S}_{(\mathrm{n})}\right) \propto(2 \pi)^{-k(n-p) / 2}|T|^{n-p / 2} \exp \left(-\frac{1}{2} \operatorname{tr} \sum_{t=p+1}^{n}\left(y(t)-\Gamma^{\prime} \mathrm{Z}(t-1)(y(t)-\right.\right.$
$\left.\Gamma^{\prime} Z t-1^{\prime}\right) T$

generally, the term (2) is very complex because the residuals $c(t)$ are nonlinear function of the coefficients $\mathrm{r}$. Moreover, to see this, let us rewrite yet), $c(t)$ and $\mathrm{r}$ as follows:

$\mathrm{y}(\mathrm{t})=\left[\begin{array}{lll}y(1, t) & y(2, t) \ldots y(k, t)\end{array}\right]^{\prime}$
$\varepsilon(\mathrm{t})=\left[\begin{array}{lll}\varepsilon(1, t) & \varepsilon(2, t) & \ldots \varepsilon(k, t)\end{array}\right]^{\prime}$

$\Gamma_{(\mathrm{k}(\mathrm{p}+\mathrm{q}) \mathrm{xk})}=\left[\begin{array}{cccc}\emptyset(1,1) & \emptyset(1,2) & \ldots & \emptyset(1, k) \\ \varnothing(2,1) & \varnothing(2,2) & \ldots & \varnothing(2, k) \\ \ldots & \ldots & & \ldots \\ \varnothing(k p, 1) & \varnothing(k p, 2) & \ldots & \varnothing(k p, k) \\ \theta(1,1) & \theta(1,2) & \ldots & \theta(1, k) \\ \theta(2,1) & \theta(2,2) & \ldots & \theta(2, k) \\ \ldots & \ldots & & \ldots \\ \theta(k q, 1) & \theta(k q, 2) & \ldots & \theta(k q, k)\end{array}\right]=\left[\begin{array}{c}\Phi_{k p x k} \\ \Theta_{k q x k}\end{array}\right]$.

Moreover, the residuals are given from the following $\mathrm{m}$ recurrence

$\varepsilon_{i}^{\prime}(\mathrm{t})=y_{i}^{\prime}(\mathrm{t})-Z_{i}^{\prime}(\mathrm{t}-1) \Gamma_{i} \quad$, where $\mathrm{i}=1,2, \ldots, \mathrm{m}$

In addition, using the g-th component of $\varepsilon_{i}(\mathrm{t})$ can be written as:

$\varepsilon(g, t)=y(g, t)$

where $g=1,2, \ldots, k$

$$
\begin{aligned}
& -\sum_{r=1}^{k} \sum_{j=1}^{p_{i}} \varepsilon(r, t-j) \Phi_{i}(r+k j-k, g) \\
& +\sum_{r=1}^{k} \sum_{i=1}^{q_{i}} \varepsilon(r, t-j) \Theta_{i}(r+k j-k, g)
\end{aligned}
$$

The recurrence (4) reasons the major problem in developing the accurate analysis of the multivariate VARMA models, however, this recurrence can be used to estimate the residuals repetition if one knows $r$ and the primary values of the residuals. That is why we need to apply the approximation approached which made by (broemeling and Shaarawy, 2014); the suggest approximation is based on replacing the exact residuals by their non-linear least squares estimates and assuming that $E(p)=$ eip -1$)=\ldots=E\left(p_{-} q_{-} 1\right)=\mathrm{O}$.

Thus, we estimate the residuals recursively by:

$$
\begin{gathered}
\varepsilon(g, t)=y(g, t)-\sum_{r=1}^{k} \sum_{r=1}^{p_{i}} \varepsilon(r, t-j) \widehat{\Phi}_{i}(r+k j-k, g) \\
+\sum_{r=1}^{k} \sum_{r=1}^{q_{i}} \varepsilon(r, t-j) \widehat{\Theta}_{i}(r+k j-k, g)
\end{gathered}
$$

Where $\mathrm{g}=1,2, \ldots \mathrm{k}$, Therefore, employ the estimates of the residuals, we can write the likelihood function approximately as:

$\mathrm{L}\left(\Gamma_{i}, \mathrm{~T} / S_{n}\right) \propto|T|^{n-p} / 2 \exp \left(-\frac{1}{2} \operatorname{tr} \sum_{t=p+1}^{n}\left(y(t)-\Gamma^{\prime} \hat{Z}(t-1)\right)\left(y^{\prime}(t)-\hat{Z}^{\prime}(t-1) \Gamma\right) T\right)$ 
Second, as a convenient selected of the

The prior density of the parameter $\mathrm{F}, \mathrm{T}$ is the following matrix normal- wishart distribution since

$$
\xi(\Gamma, T) \alpha \xi_{1}(\Gamma / T) \xi_{2}(T)
$$

where

$\xi_{1}(\Gamma / T) \alpha|T|^{k(p+q) / 2} \exp \left(-\frac{1}{2} \operatorname{tr}(\Gamma-\widehat{\Gamma})^{\prime} V(\Gamma-\widehat{\Gamma}) T\right)$

And, $\xi_{2}(T) \alpha|T|^{\frac{1}{2}(a-(k+1))} \exp \operatorname{Tr}\left(-\frac{1}{2} M T\right)$,Then we can write $\xi(\Gamma, \mathrm{T})$ as next:

$\xi(\Gamma, \mathrm{T}) \alpha|T|^{\frac{1}{2}(k(p+q)+(a-(k+1))} \exp -\frac{1}{2} \operatorname{Tr}\left(\left((\Gamma-D)^{\prime} V(\Gamma-D)+M\right) T\right)$

As a prior distribution for a natural, conjugate distribution.

If the one cannot or is unwilling to specify the hyper-parameters $\widehat{\Theta}, V$,

$\mathrm{M} r$ then we can use the jefferys vague prior as following:

$f(T)=|T|^{\frac{1}{2}(K+1)}$

Last, we will need to have a prior probability mass function of the order q, which can be written as:

$\beta \mathrm{i}=\operatorname{Pr}(\mathrm{q}=\mathrm{i}) \quad, \mathrm{i}=1,2, \ldots \ldots ., Q$

Theroy :

Combining the approximation conditional likelihood function in (4) with the priors (5) and (7), the approximation marginal posterior mass function of order q, the order of VARMA model has form :

$h\left(p, q / S_{(n)}\right) \alpha \beta_{i}\left|A_{q}\right|^{-K / 2}\left|V_{q}\right|^{k / 2}\left|C_{q}\right|^{-\frac{1}{2}[n+a]} \prod_{j=1}^{k} \Gamma\left(\frac{[n+a+j-k(p+q)]}{2}\right)$

Since

$\mathrm{L}\left(\Gamma, p, q, \mathrm{~T} / S_{(n)}\right) \propto \beta_{i}|T|^{\frac{n-p+k(p+q)+a-(k+1)}{2}} \exp \left(-\frac{1}{2} \operatorname{tr}\left\{(\Gamma-D)^{\prime} V(\Gamma-D)+M+\right.\right.$

$\left.\sum_{t=q+1}^{n}\left(y(t)-\Gamma^{\prime} Z(t-1) T\left(y(t)-\Gamma^{\prime} Z(t-1)^{\prime}\right)\right\} \mathrm{T}\right)$

Then we can re-write the last equation as

next:L( $\left(\Gamma, p, q, \mathrm{~T} / S_{(n)}\right) \alpha \beta_{i}|T|^{\frac{n-p+k(p+q)+a-(k+1)}{2}} \exp \left(-\frac{1}{2} \operatorname{tr}\left\{(\Gamma-D)^{\prime} V(\Gamma-D)+M+\right.\right.$

$\left.\sum_{t=p+1}^{n}\left(y(t)-\Gamma^{\prime} Z(t-1) T\left(y(t)-\Gamma^{\prime} Z(t-1)^{\prime}\right)\right\} \mathrm{T}\right)$

$\mathrm{L}\left(\Gamma, p, q, \mathrm{~T} / S_{(n)}\right) \alpha \beta_{i}|T|^{W(\Gamma) / 2} \exp \left(-\frac{1}{2} \operatorname{tr}\left\{(\Gamma-D)^{\prime} V(\Gamma-D)+M+\sum_{t=p+1}^{n}(y(t)-\right.\right.$

$\left.\left.\Gamma^{\prime} Z(t-1) T\left(y(t)-\Gamma^{\prime} Z(t-1)^{\prime}\right)\right\} \mathrm{T}\right)$

If $\mathrm{w}(\Gamma)=\mathrm{n}-\mathrm{p}+\mathrm{k}(\mathrm{p}+\mathrm{g})+\mathrm{a}-(\mathrm{k}+1)$

The term between \{\} in the exponent of equation (11) can be written as:

$\left\{\Gamma^{\prime}\left[V+\sum_{t=p+1}^{n} Z(t-1) Z^{\prime}(t-1)\right] \Gamma-\Gamma^{\prime}\left[\mathrm{VD}+\sum_{t=p+1}^{n} Z(t-1) y^{\prime}(t)\right]-\left[D^{\prime} V+\right.\right.$ $\left.\left.\sum_{t=p+1}^{n} y(t) Z^{\prime}(t-1)\right] \Gamma+D^{\prime} V D+M+\sum_{t=p+1}^{n} y(t) y^{\prime}(t)\right\}$

By completing the square on I', the last form can be also re-written as next:

$=\left[\Gamma-A^{-1} \mathrm{~B}\right]^{\prime} A\left[\Gamma-A^{-1} \mathrm{~B}\right]+\mathrm{C}$ 


$$
\begin{aligned}
& \text { Where, } \\
& A=V+\sum_{t=p+1}^{n} Z(t-1) Z^{\prime}(t-1) \\
& B=V D+\sum_{t=p+1}^{n} Z(t-1) y^{\prime}(t) \\
& \text { And } \\
& C=D^{\prime} V D+M+\sum_{t=p+1}^{n} y(t) y^{\prime}(t)-B^{\prime} A^{-1} B
\end{aligned}
$$

Substitute from (14) in (11), we find that:

$\mathrm{L}\left(\Gamma, p, q, \mathrm{~T} / S_{(n)}\right) \alpha \beta_{i}|T|^{W(\Gamma) / 2} \exp \left(-\frac{1}{2} \operatorname{tr} C T\right) * \exp \left(-\frac{1}{2} \operatorname{tr}\left[\Gamma-A^{-1} B\right]^{\prime} A\left[\Gamma-A^{-1} \mathrm{~B}\right] T\right) .$.

Since,

$\int_{-\infty}^{\infty} \ldots \int_{-\infty}^{\infty} \exp \left(-\frac{1}{2}(x-\eta)^{\prime} C^{-1}(x-\eta) d x_{1} \ldots d x_{n}=(\sqrt{2 \pi})^{n}|C|^{\frac{1}{2}}\right.$

then; Integrating (18) consideration to $\Gamma$, we can have a joint posterior distribution of $\mathrm{p}, \mathrm{q}$ and $\mathrm{T}$ as next:

$g_{1}\left(p, q, T / S_{(n)}\right) \alpha \beta_{i}|T|^{\frac{W(\Gamma)}{2}} \exp \left(-\frac{1}{2} \operatorname{tr} C T\right) *(2 \pi)^{-(p+q) k^{2} / 2}|A|^{-k / 2}|T|^{-(p+q) k / 2}$

It might be refer that the integral of (18) with consideration to $r$ is done employ the matrix normal complete. However, (19) could be written as next:

$g_{1}\left(p, q, T / S_{(n)}\right) \alpha \beta_{i}(2 \pi)^{\frac{(p+q) k}{2}}|A|^{-\frac{k}{2}}|\mathrm{~V}|^{\frac{k}{2}}|\mathrm{~T}|^{\frac{1}{2}[W(\Gamma)-K(p+q)+2]-1} * \exp \left(-\frac{1}{2} \operatorname{tr} C T\right)$.

In addition, since

$\int_{T>0}|T|^{\frac{1}{2} q-1} \exp \left(-\frac{1}{2} \operatorname{tr} T B\right) d T=|B|^{-\frac{1}{2}(q+k-1)} 2^{\frac{1}{2} k(q+k-1)} \Gamma_{k}\left(\frac{q+k-1}{2}\right)$

then Integrating (20) regard to $\mathrm{T}$ we can have a joint posterior distribution of $\mathrm{p}$ and $\mathrm{q}$, which is the marginal posterior mass function as next:

$$
\begin{gathered}
h\left(p, q / S_{(n)}\right) \alpha \quad \beta_{i}(2 \pi)^{\frac{(p+q) k}{2}}|A|^{-\frac{k}{2}}|V|^{\frac{k}{2}}|C|^{-\frac{1}{2}\left[q_{p+q}+k-1\right]} 2^{\frac{1}{2} k\left[q_{p+q}+k-1\right]} * \\
\quad \Gamma_{k}\left(\frac{\left[q_{p+q}+k-1\right]}{2}\right.
\end{gathered}
$$

Where, $\mathrm{q}_{\mathrm{p}}+_{\mathrm{q}}=\mathrm{w}(\Gamma)-\mathrm{k}(\mathrm{p}+\mathrm{q})+2$ and $\Gamma_{\mathrm{k}}$ is a generalized gamma function, (Box and Tiao, 1973).

The last equation (3.19)/ can be written as next:

$h\left(p, q / S_{(n)}\right) \alpha \quad \beta_{i}|A|^{-\frac{k}{2}}|V|^{\frac{k}{2}}|C|^{-\frac{1}{2}\left[q_{p+q}+k-1\right]} 2^{\frac{k q_{p+q}}{2}} * \Pi_{j=1}^{k} \Gamma\left(\frac{\left[q_{p+q}+j-1\right]}{2}\right)$

Finally, with direct substitution of qp+Q' we get the next form:

$$
h\left(p, q / S_{(n)}\right) \alpha \beta_{i}|A|^{-\frac{k}{2}}|V|^{\frac{k}{2}}|C|^{-\frac{1}{2}[n-p+a]} \quad \Pi_{j=1}^{k} \Gamma\left(\frac{[n-p+a+j-k]}{2}\right)
$$

While, $\mathrm{n}-\mathrm{p}+\mathrm{a}-\mathrm{k}>\mathrm{o}$.

Corollary :

Combining the approximation conditional likelihood function in (4) with the Jeffrey's priors (6), the approximation marginal posterior mass function of order q, the order of VARMA model has the form:

$$
h_{1}\left(q / S_{(n)}\right) \alpha\left|A^{*}\right|^{-\frac{k}{2}}\left|C_{q}\right|^{-\frac{1}{2}[n-p-(p+q) k]} \Pi_{j=1}^{k} \Gamma\left(\frac{[n-p-k-k(p+q)+j]}{2}\right)
$$


If the one has a little information about the parameters and wants to use the Jeffrey's vague prior, one will have the following posterior mass function of the order $\mathrm{q}$, since

With following, the same procedure which has been developed in theorem

Where:

$$
\begin{aligned}
& A^{*}=\sum_{t=p+1}^{n} Z(t-1) Z^{\prime}(t-1) \\
& B^{*}=\sum_{t=p+1}^{n} Z(t-1) Y^{\prime}(t) \text { هنا الدعادلة اكتب . هنا المعادلة اكتب . } \\
& C=\sum_{t=p+1}^{n} Y(t) Y^{\prime}(t)-B^{*^{\prime}} A^{*-1} B^{*}
\end{aligned}
$$

We will reach to next as:

$$
h_{1}\left(p, q / S_{(n)}\right) \alpha\left|A^{*}\right|^{-\frac{k}{2}}|C|^{-\frac{1}{2}[n-p+(p+q) k]} \Pi_{j=1}^{k} \Gamma\left(\frac{[n-p-k-k(p+q)+j]}{2}\right)
$$

While, $\mathrm{n}-\mathrm{p}-\mathrm{k}-\mathrm{kp}+1>0$.

The last two forms (24) and (25) are the forms of the posterior mass function, which is a suitable and easy to mange it with a computer. Then one may inspect the plot of the posterior mass function over the grid of the $\mathrm{P}$ and $\mathrm{Q}$ and pick out the value of the $\mathrm{p}$ and $\mathrm{q}$ at which the posterior mass function attains its maximum to be the identified the order of the time series data being analyzed.

\section{REFERENCES}

(1) Ali, M.M.(2017). "Analysis of autoregressive moving average models: estimation and prediction." Biometric, Vol. 64, p. 535-545.

(2) Ansley, G. F. (2019). "An algorithm for the exact likelihood of mixed autoregressive moving average process. " Biometrika, Vol. 66, P.59- 65.

(3) Ansley, C. F. and Kohn, R. (2013). "Exact Likelihood Of Vector Autoregressive Moving Average Process With Missing or Aggregated Data", Biometrika, 70, pp. 275-278.

(4) Akiake, H. (2019). Fitting Autoregressive models for prediction. Annals of institute of statistical mathematics, Vol. 21, pp. 243- 247.

(5) Box, G. and Jenkins, G. (2016). Time series analysis, forecasting and control. $2^{\text {nd }}$ Edition, Holden-day, San Francisco.

(6) Box, G. and Tiao, G (2013). Bayesian Inference in Statistical Analysis.

Addison Wesley, Massachusetts.

(7) Broemeling, L. and Shaarawy, S. (2019) Time series: A Bayesian Inference and forecasting with Multiple Autoregressive moving

average models, communication in statistics, Institute of statistical studies and research, Cairo, Egypt, VoI.2,PP. 14811509.

(8) Diaz, J. and Farah, J (2011). Bayesian identification of Autoregressive process 
presented at $22^{\text {nd }}$ NBER-NSC. Seminar on Bayesian inference in Econometrics.

(9) Hillmer, Steven C. and Tiao, George c., (2019). "likelihood Function of a stationary multiple autoregressive moving average model", Journal of the American statistical association, Vol. 74, No. 367, pp. 652- 660.

(10) lutkepohl, H. (2013). Introduction to multiple time series analysis, Second edition, Springer - Verlag Berlin.

(11) Newbold, P. (2013). Bayesian Estimation of Box and Jenkins Transfer Function Model for noise models. Journal of royal statistical Guide Society, Series B, Vol. 35, No.2, pp. 323336.

(12) Shaarawy, S., Ali, S. (2013). Bayesian Identification of seasonal autoregressive models. Commune. Statist. Theory. Meth. 32:10671084.

(13) Shaarawy, S., Ali, S. (2017). Bayesian Identification of Multivariate autoregressive Process. Commune. Statist. Theory. Meth. 37:791-802.

(14) Schwartz, G. (2018). Estimating the dimension of a model. Annals of Statistics 6(2), 461-464

(15) Zellner, A.,2011,anIntroduction to Bayesian Inferencein Econometrics. New York 